\title{
Impact of Continuous Renal Replacement Therapy as Treatment for Sepsis-Associated Acute Kidney Injury on Lactate Levels and the Risk of 28-Day Mortality in Intensive Care Units
}

\section{Luming Zhang}

The First Affiliated Hospital of Jinan University

\section{Zichen Wang}

California Department of Public Health

\section{Fengshuo Xu}

The First Affiliated Hospital of Jinan University

\section{Yinlong Ren}

The First Affiliated Hospital of Jinan University

Didi Han

The First Affiliated Hospital of Jinan University

\section{Chengzhuo Li}

The First Affiliated Hospital of Jinan University

Jun Lyu

The First Affiliated Hospital of Jinan University

Haiyan Yin ( $\nabla$ yinhaiyan1867@126.com )

The First Affiliated Hospital of Jinan University https://orcid.org/0000-0002-9680-4219

\section{Research}

Keywords: Continuous Renal Replacement Therapy, Sepsis, Acute Kidney Injury, Lactate, Joint Model

Posted Date: January 26th, 2021

DOI: https://doi.org/10.21203/rs.3.rs-152669/v1

License: @ (i) This work is licensed under a Creative Commons Attribution 4.0 International License. Read Full License 


\section{Abstract}

Background: Sepsis has high incidence and fatality rates in intensive care units, often leading to renal failure. The effectiveness of continuous renal replacement therapy (CRRT) in sepsis-associated acute kidney injury (S-AKI) patients is currently uncertain.

Aim: Joint mode was used to determine the effect of CRRT on the lactate levels and survival of S-AKI patients.

Methods: A retrospective study was applied to patients with sepsis and AKI, which were extracted from the MIMIC-III public database, with the endpoint being 28-day mortality. Every lactate level measurement within 28 days was observed and calculated using logarithms. Joint model combined the longitudinal analysis of the natural logarithm of the lactate level [log(lactate)] in longitudinal submodel and Cox regression by trajectory function, demonstrating the effects of CRRT on 28day survival and log(lactate) changes, and its final relationship with the event status.

Results: Among the 717 S-AKI patients, 157 received CRRT. CRRT was not associated with 28-day mortality. After adjustments, the relationship between CRRT use and log(lactate) elevation was statistically significant. The parameter estimation of CRRT and log(lactate) indicated that using CRRT will increase log(lactate) by 0.041 in S-AKI patients. The joint model also instigated a fixed association between changes in the lactate level and the event result, revealing an exp value of $(1.755)=5.78$, indicating that an increase of one unit in log(lactate) will increase the risk of 28-day mortality 5.78fold.

Conclusion: CRRT does not improve the prognosis of patients with sepsis and acute kidney injury in critical-care units and has a tendency of increasing lactate levels, which is a significant risk factor for the prognosis.

\section{Introduction}

Sepsis is a common clinical critical illness ${ }^{1}$ that causes dysfunction of the immune and blood coagulation systems, and affected patients usually have insufficient tissue perfusion ${ }^{2}$. As a product of glycolysis, lactate capacity directly reflects the degree of anaerobic glycolysis in tissues and cells, and is therefore a commonly used clinical indicator that effectively reflects tissue hypoxia and hypoperfusion. It has also been shown to be closely related to the prognosis of sepsis patients ${ }^{3}$. Several studies have shown that sepsis patients can have increased lactate levels due to low clearance or liver and kidney dysfunction ${ }^{4}$.

Sepsis is also characterized by persistent refractory hypotension, hyperlactic acidemia, and organ dysfunction after aggressive fluid resuscitation ${ }^{2}$. Studies have indicated that sepsis can often lead to acute kidney injury (AKI). Up to $50 \%$ of sepsis patients develop $\mathrm{AKI}$, leading to poor prognosis and a mortality rate $(75 \%)^{5,6}$ that is significantly higher than that in sepsis patients without organ failure ${ }^{7}$. Continuous renal replacement therapy (CRRT) is a method of extracorporeal blood purification. Current clinical practices consider CRRT to able to maintain internal environment stability by removing toxic substances from the kidneys, and also supports organs function. CRRT has recently been widely used in sepsis treatment ${ }^{7,8}$. However, the efficacy of CRRT in S-AKI patients remains uncertain. Studies have indicated that lactate levels and the 24-hour lactate clearance rate after CRRT use are related to the 28-day mortality of S-AKI patients ${ }^{9}$, while CRRT with massive hemofiltration is ineffective for severe lactic acidosis ${ }^{10,11}$.

A joint model (JM) technique was used in this study to investigate the influence of CRRT on lactate levels and its efficacy on S-AKI patient survival. Applying a JM to longitudinal and event time data has become a valuable follow-up data analysis method that combines the linear mixed model for longitudinal data and the Cox proportional-hazards model for time-to-event data by trajectory function ${ }^{12}$. A JM provides a more effective method for predicting the impact of treatments 
on outcomes, a more effective estimation of treatment effects on longitudinal data, and reduces the bias of the overall prediction when compared to the single linear mixed model and the Cox proportional-hazards model ${ }^{12,13}$.

\section{Methods}

\section{Data source and extraction}

Data were extracted from the Medical Information Mart for Intensive Care-III (MIMIC-III) database, which is a public database containing data on over 50,000 patients in critical-care units ${ }^{14}$. SQL was used to extract data before further analysis. The data extracted were the natural logarithm of the lactate level [log(lactate)], CRRT use, ventilator use, vasopressor use, demographic data including age, sex, and race, vital signs including mean blood pressure, respiration rate (RR), heart rate, skin temperature, oxygen saturation, and partial pressure of carbon dioxide, laboratory test results including urine output (UO), pH, albumin, bicarbonate, creatinine (CR), glucose, magnesium (MG), phosphorus (PHOS), potassium, sodium (NA), hematocrit, hemoglobin, platelet, red blood cell width (RDW), white blood cells, neutrophil/lymphocyte ratio (NLR), comorbidities including heart failure, hypertension, liver diseases, fluid-electrolyte imbalance, and SOFA score.

\section{Study population}

This study selected 1295 S-AKI patients from the MIMIC-III database. The inclusion criteria included meeting Sepsis-3 criteria $^{15}$, since they have severe infection and organ failure (SOFA score $\geq 2$ ); AKI that occurred during hospitalization (increase in SCr by $0.3 \mathrm{mg} / \mathrm{dl}$ [6.5 $\mathrm{mmol} / \mathrm{l}]$ ) within 48 hours; a 1.5-fold increase in SCr from baseline, which is known or presumed to have occurred within the previous 7 days; urine volume $<0.5 \mathrm{ml} / \mathrm{kg} /$ hour for 6 hours ${ }^{16}$. Patients younger than 18 years and without longitudinal records of lactate levels within 28 days were excluded, leaving 717 patients as the study population.

\section{Statistical analysis}

\section{Longitudinal data analysis}

Every lactate level measurement made within 28 days in this study was observed and assessed using logarithms. Linear mixed-effect models were used to analyze longitudinal data. The dependent variable was log(lactate). The independent variables were CRRT use, time of lactate level, and their two-way interaction, NLR, SOFA score group (dichotomized into $<6$ and $\geq 6$ ), and age.

\section{Time-to-event data analyses}

Time-to-event data were first analyzed using the Cox proportional-hazards model to determine the relationship between CRRT and 28-day survival. Variables were screened using multivariable regression if they differed significantly between the CRRT and non-CRRT groups.

\section{Joint model}

The JM combined the longitudinal analysis of log(lactate) and Cox regression using a trajectory function, revealing the effect of CRRT on 28-day survival as well as log(lactate) changes and their relationship with the event status (Fig. 1).

All statistical analyses were performed using R software, with the JM being constructed using the "JM" package.

Continuous variables were presented as medians with normality-based quartiles, with $\mathrm{p}$ values calculated using Student's t-tests. Categorical variables were presented as numbers and percentages, with $p$ values calculated using chi-square tests.

\section{Results}




\section{Patient characteristics}

Among the 717 S-AKI patients, 157 received CRRT. Table 1 lists the baseline characteristics of the two CRRT groups including demographics, vital signs, laboratory-result comorbidities, and SOFA scores. These characteristics demonstrate that CRRT was associated with significant differences in age, race, RDW, NA, MG, PHOS, CR, and UO, liver disease status, ventilator and vasopressor use, SOFA score, and 28-day mortality.

The longitudinal lactate data of 3661 observations were displayed using trajectory functions and plotted using interaction figures. Figure 2 indicates the linear trajectory record of lactate and log(lactate) for each patient over the 28-day analysis period. The figure shows that most observations were concentrated within the first 5 days after patient admission. Lactate levels of patients ranged from 0.5 to 20 , with log(lactate) ranging from -0.4 to 1.3. Cox regression was performed to analyze the relationship between CRRT and 28-day survival, with CRRT being adjusted for by age, race, RDW, NA, MG, PHOS, CR, and UO, liver disease status, ventilator and vasopressor use, and SOFA score. The Schoenfeld residuals test (Fig. 3) was used to determine the independence of residuals and the time to test for the proportional-hazards hypothesis in the Cox model. The results in Fig. 3 revealed that CRRT was not a time-dependent variable and therefore could be analyzed directly using Cox regression. CRRT was also found to not be related to 28-day survival in S-AKI patients after adjusting multiple variables.

Linear mixed-effect models, on the other hand, indicated the association between CRRT and log(lactate). Among the independent variables, CRRT and lactate observation time were statistically correlated with log(lactate) in longitudinal status. The result of the JM demonstrated that after combining the longitudinal submodel of lactate and the survival model of CRRT, CRRT was still not related to the 28-day survival of S-AKI patients. After adjusting the survival submodel, the correlation between CRRT use and elevation of log(lactate) was statistically significant. The parameter estimation of CRRT and log(lactate) indicated that using CRRT increased log(lactate) by 0.041 in S-AKI patients. The JM also instigated a fixed association between lactate level changes and the event result, producing an exp value of $(1.755)=5.78$, indicating that an increase of one unit of log(lactate) will increase the risk of 28-day mortality 5.78-fold (Table 2).

\section{Discussion}

Our survival submodel indicates that CRRT use has no effect on the 28-day mortality of S-AKI patients in critical care. SAKI patient often have weaker glomerular filtration functions, which can increase the probability of adverse symptoms such as electrolyte/acidolysis imbalance and also impair metabolism ${ }^{17}$. Therefore, preventing further aggravation through eliminating metabolic toxins to prevent further aggravation is necessary. CRRT is currently one of the main treatment methods ${ }^{18}$. This intervention focuses on replacing kidney function, removing toxic substances such as $\mathrm{CR}$, or improving the fluid-electrolyte imbalance that allows for future treatments since patients are in a stable condition ${ }^{18-20}$. Although CRRT is still the most common treatment for S-AKI, studies have indicated that renal replacement therapy cannot improve its survival ${ }^{8,21}$. Whether to apply high-intensity CRRT to S-AKI patients remains controversial. Some studies have indicated that the use of high-dose renal replacement therapy does not improve the overall survival of patients or the recovery of renal function ${ }^{21,22}$. The efficacy of CRRT on the prognosis of S-AKI can therefore not be confirmed.

The analysis of longitudinal submodel indicated that the use of CRRT is associated with increases in lactate levels within 28 days, while the joint modeling of longitudinal and survival data indicated that lactate level changes were associated with mortality and that $\log$ (lactate) is a risk factor for 28-day mortality in S-AKI patients. Many studies have confirmed lactate as a powerful biomarker for sepsis with renal damage and can accurately predict mortality ${ }^{23-25}$. Reducing lactate is therefore a vital procedure for improving the likelihood of patient survival. However, although CRRT is the most popular treatment in patients with sepsis and AKI, there is still a possibility that it cannot reduce acidosis, resulting in the continued elevation of lactate levels after CRRT is performed ${ }^{26}$. Potential explanations include how in clinical practice, since it is 
generally believed that if lactate fluctuates to a lower range, there will be no negative impact on the prognosis of patients. Therefore, these lower lactate levels may not receive adequate attention. Additionally, lactate levels may be affected by the buffer used in $\mathrm{CRRT}^{27}$. For example, when using lactate-based fluids, sepsis patients failed to completely metabolize lactate ${ }^{28}$, potentially leading to increased lactate levels, eventually developing into metabolic acidosis hyperlactatemia. Finally, when sepsis patients with AKI have more serious infections, vascular permeability is increased and the responses to vasopressors are poor. Although CRRT can temporarily remove toxins and other substances from the body, circulatory ischemia and hypoxia still cannot be improved, and lactate will also continuously increase ${ }^{29}$. Our research found that across all ranges of lactate levels, the risk of death increased 5.78-fold for every unit increase in log(lactate). When using CRRT to treat patients with sepsis and AKI, we should therefore pay more attention to changes in patient lactate levels.

This study had some limitations. Some factors during the use of CRRT will impact survival, such as filter coagulation, the conversion of patients to intermittent hemodialysis after hemodynamic stability is reached, and the death of patients during treatment. The limited database means we could not analyze these impacts retrospectively. Additionally, the singlecenter nature of the database reduces the generalizability of our results, which must therefore be tested in further research.

\section{Conclusion}

CRRT is not an effective treatment for S-AKI patients in the critical-care unit, and has a tendency to increase lactate levels, which is a significant risk factor for the prognosis. Other treatments that focus on controlling lactate levels should receive more attention in critical care.

\section{Declarations}

\section{Ethics approval and Consent to participate}

The study was an analysis of a third-party anonymized publicly available database with pre-existing institutional review board (IRB) approval.

\section{Consent for publication}

Not applicable

\section{Availability of supporting data}

The data were available on the MIMIC-III website at https://mimic.physionet.org/

\section{Competing interests}

The authors declare that they have no competing interests.

\section{Funding}

This work was supported by the National Natural Science Foundation of China (No. 82072232; 81871585), the Natural Science Foundation of Guangdong Province (No. 2018A030313058), Technology and Innovation Commission of Guangzhou Science, China (No.201804010308).

\section{Authors' contributions}

LZ created the study protocol, performed the statistical analyses and wrote the first manuscript draft. ZW conceived the study and critically revised the manuscript. FX assisted with the study design and performed data collection. YR assisted with data collection and manuscript editing. DH confirmed the data and assisted with the statistical analyses. CL assisted 
with study coordination and helped draft the manuscript. JL assisted with manuscript revision and data confirmation. HY contributed to data interpretation and manuscript revision. All authors read and approved the final manuscript.

\section{Acknowledgment}

None

\section{References}

1. Bracht $H$, Hafner S, Weiß M. [Sepsis Update: Definition and Epidemiology]. Anasthesiol Intensivmed Notfallmed Schmerzther. 2019;54(1):10-20. doi:10.1055/a-0625-5492.

2. Cecconi M. Evans, et al. Sepsis and septic shock. Lancet. 2018.

3. Nolt B, Tu F, Wang X, et al. Lactate and Immunosuppression in Sepsis. Shock. 2018;49(2):120-5. doi:10.1097/SHK.0000000000000958.

4. Chertoff J, Chisum M, Garcia B, Lascano J. Lactate kinetics in sepsis and septic shock: a review of the literature and rationale for further research. J intensive care. 2015;3:39. doi:10.1186/s40560-015-0105-4.

5. Poston JT, Koyner JL. Sepsis associated acute kidney injury. BMJ. 2019;364:k4891. doi:10.1136/bmj.k4891.

6. Bagshaw SM, George C, Bellomo R, Committee ADM. Early acute kidney injury and sepsis: a multicentre evaluation. Crit Care. 2008;12(2):R47-7. doi:10.1186/cc6863.

7. Pozzato M, Livigni S, Roccatello D. [Extracorporeal therapy in sepsis]. G Ital di Nefrol organo Uff della Soc Ital di Nefrol. 2019;36(2).

8. Romagnoli S, Ricci Z, Ronco C. CRRT for sepsis-induced acute kidney injury. Curr Opin Crit Care. 2018;24(6). https://journals.Iww.com/cocriticalcare/Fulltext/2018/12000/CRRT_for_sepsis_induced_acute_kidney_injury.10.aspx.

9. Passos RH, Ramos JGR, Gobatto A, et al. Lactate clearance is associated with mortality in septic patients with acute kidney injury requiring continuous renal replacement therapy: A cohort study. Med (Baltim). 2016;95(40):e5112. doi:10.1097/MD.0000000000005112.

10. Cheungpasitporn W, Zand L, Dillon JJ, Qian Q, Leung N. Lactate clearance and metabolic aspects of continuous highvolume hemofiltration. Clin Kidney J. 2015;8(4):374-7. doi:10.1093/ckj/sfv045.

11. Liu Y, Ouyang B, Chen J, et al. Effects of different doses in continuous veno-venous hemofiltration on plasma lactate in critically ill patients. Chin Med J (Engl). 2014;127(10):1827-32.

12. Ibrahim JG, Chu H, Chen LM. Basic concepts and methods for joint models of longitudinal and survival data. J Clin Oncol. 2010;28(16):2796-801. doi:10.1200/JC0.2009.25.0654.

13. Commenges D, Jacqmin-Gadda H. - Joint Models for Longitudinal and Time-to-Event Data.; 2020. doi:10.1201/b19109-17.

14. Johnson AEW, Pollard TJ, Shen L, et al. MIMIC-III, a freely accessible critical care database. entific Data.

15. Singer M, Deutschman CS, Seymour CW, et al. The Third International Consensus Definitions for Sepsis and Septic Shock (Sepsis-3). JAMA. 2016;315(8):801-10. doi:10.1001/jama.2016.0287.

16. Kellum JA, Lameire N. Diagnosis, evaluation, and management of acute kidney injury: a KDIGO summary (Part 1). Crit Care. 2013;17(1):204. doi:10.1186/cc11454.

17. Regueira T, Andresen M, Mercado M, Downey P. [Physiopathology of acute renal failure during sepsis]. Med intensiva. 2011;35(7):424-32. doi:10.1016/j.medin.2011.03.011.

18. Prowle JR, Schneider A, Bellomo R. Clinical review: Optimal dose of continuous renal replacement therapy in acute kidney injury. Crit Care. 2011;15(2):207. doi:10.1186/cc9415. 
19. Michael J. THE CLINICAL APPLICATION OF CRRT-CURRENT STATUS: Continuous Renal Replacement Therapy in Sepsis and Multisystem Organ Failure. Semin Dial. 2009.

20. Zhang J, Tian J, Sun H, et al. How Does Continuous Renal Replacement Therapy Affect Septic Acute Kidney Injury? Blood Purif. 2018;46(4):326-31. doi:10.1159/000492026.

21. Van Wert R, Friedrich JO, Scales DC, Wald R, Adhikari NKJ. Group for the U of TAKIR. High-dose renal replacement therapy for acute kidney injury: Systematic review and meta-analysis. Crit Care Med. 2010;38(5). https://journals.Iww.com/ccmjournal/Fulltext/2010/05000/High_dose_renal_replacement_therapy_for_acute.18.aspx.

22. Li P, Qu L, Qi D, et al. High-dose versus low-dose haemofiltration for the treatment of critically ill patients with acute kidney injury: an updated systematic review and meta-analysis. BMJ Open. 2017;7(10):e014171. doi:10.1136/bmjopen-2016-014171.

23. Hsu Y-C, Hsu C-W. Septic acute kidney injury patients in emergency department: The risk factors and its correlation to serum lactate. Am J Emerg Med. 2019;37(2):204-8. doi:10.1016/j.ajem.2018.05.012.

24. Serum lactates and. acute kidney injury in patients with sepsis: A cohort analysis. Cogent Med. 2017;4(1).

25. Aliustaoglu Bayar AE, Aksay E, Oray NC. Lactate measurements accurately predicts 1-week mortality in emergency department patients with acute kidney injury. Turkish J Emerg Med. 2019;19(4):136-40. doi:https://doi.org/10.1016/j.tjem.2019.08.002.

26. Page B, Vieillard-Baron A, Chergui K, et al. Early veno-venous haemodiafiltration for sepsis-related multiple organ failure. Crit Care. 2005;9(6):R755-63. doi:10.1186/cc3886.

27. Morgera S, Heering P, Szentandrasi T, et al. Comparison of a lactate-versus acetate-based hemofiltration replacement fluid in patients with acute renal failure. Ren Fail. 1997;19(1):155-64. doi:10.3109/08860229709026270.

28. Davenport A, Will EJ, Davison AM. The effect of lactate-buffered solutions on the acid-base status of patients with renal failure. Nephrol Dial Transplant Off Publ Eur Dial Transpl Assoc - Eur Ren Assoc. 1989;4(9):800-4.

29. Jacobi J. Pathophysiology of sepsis. Am J Heal Pharm AJHP Off J Am Soc Heal Pharm. 2002;59(Suppl 1):3-8. doi:10.1093/ajhp/59.suppl_1.S3.

\section{Tables}

Table.1 Baseline characteristics between CRRT Unreceived /Received Group 


\begin{tabular}{|c|c|c|c|}
\hline Characteristics & CRRT Unreceived & CRRT Received & $p$ value \\
\hline $\mathrm{N}$ & 560 & 157 & \\
\hline Age & $71.0(61.0,81.0)$ & $67.0(58.0,77.0)$ & $0.001^{*}$ \\
\hline Sex $n,(\%)$ & & & 0.459 \\
\hline Male & $347(62.0)$ & 103(65.6) & \\
\hline Female & 213(38.0) & $54(34.4)$ & \\
\hline Ethnicity n,(\%) & & & $0.013^{\star}$ \\
\hline White & $408(72.9)$ & $109(69.4)$ & \\
\hline Black & $85(15.2)$ & $16(10.2)$ & \\
\hline Others & $67(12.0)$ & $32(20.4)$ & \\
\hline \multicolumn{4}{|l|}{ Vital signs } \\
\hline Mean blood pressure (mmHg) & $70.3(65.1,75.6)$ & $68.4(64.1,74.6)$ & 0.057 \\
\hline Heart rate (min- 1$)$ & $84.0(73.9,98.2)$ & $86.9(76.8,97.3)$ & 0.271 \\
\hline Respiratory rate (min-1) & 19.7(17.4,22.9) & $19.9(17.4,22.8)$ & 0.633 \\
\hline Oxygen saturation (\%) & $97.5(96.2,98.9)$ & $97.2(96.0,98.7)$ & 0.119 \\
\hline Skin temperature $\left({ }^{\circ} \mathrm{C}\right)$ & $36.7(36.2,37.2)$ & $36.7(36.2,37.3)$ & 0.906 \\
\hline Partial pressure of carbon dioxide $(\mathrm{mmHg})$ & $39.0(33.0,48.0)$ & $42.0(36.0,48.0)$ & 0.091 \\
\hline \multicolumn{4}{|l|}{ Laboratory result } \\
\hline White Blood Cell (k/uL) & $11.80(7.40,17.12)$ & $11.70(7.40,16.80)$ & 0.850 \\
\hline Neutrophil/lymphocyte ratio & $11.86(5.90,21.80)$ & $11.11(6.80,21.50)$ & 0.563 \\
\hline Hemoglobin $(\mathrm{g} / \mathrm{dL})$ & $10.60(9.40,12.10)$ & $10.70(9.30,11.90)$ & 0.731 \\
\hline Red blood Cell Distribution Width (\%) & $16.25(14.80,18.20)$ & $16.80(15.50,18.40)$ & $0.023^{*}$ \\
\hline Hematocrit (\%) & $32.60(28.90,37.00)$ & $32.60(28.50,37.40)$ & 0.999 \\
\hline Platelet (K/uL) & $194.00(126.25,272.25)$ & $196.00(120.00,271.00)$ & 0.972 \\
\hline Sodium (mEq/L) & $138.00(134.00,141.00)$ & $137.00(133.00,140.00)$ & $0.017^{\star}$ \\
\hline Potassium (mEq/L) & $4.50(4.00,5.20)$ & $4.60(4.00,5.30)$ & 0.320 \\
\hline Magnesium (mg/dL) & $2.00(1.70,2.30)$ & $2.00(1.80,2.40)$ & $0.034^{*}$ \\
\hline Bicarbonate(mEq/L) & $22.0(19.0,26.3)$ & $22.0(18.0,26.0)$ & 0.526 \\
\hline Phosphate(mg/dL) & $3.90(3.00,5.00)$ & $4.60(3.50,6.20)$ & $<0.001^{*}$ \\
\hline Albumin(mg/dL) & $2.9(2.5,3.3)$ & $2.8(2.4,3.3)$ & 0.629 \\
\hline Glucose(mg/dL) & $128.00(102.00,179.25)$ & $126.00(98.00,175.00)$ & 0.789 \\
\hline
\end{tabular}




\begin{tabular}{|llll|}
\hline Creatinine $(\mathrm{K} / \mathrm{uL})$ & $2.70(1.87,4.20)$ & $3.70(2.70,5.20)$ & $<0.001^{*}$ \\
\hline $\mathrm{PH}$ & $7.4(7.3,7.4)$ & $7.3(7.3,7.4)$ & 0.061 \\
\hline Urine output $(\mathrm{mL})$ & $804.0(258.5,1459.4)$ & $195.0(39.0,602.0)$ & $<0.001^{*}$ \\
\hline Comorbidities, $\mathrm{n}(\%)$ & & & 0.624 \\
\hline Congestive heart failure & $310(55.4)$ & $91(58.0)$ & 0.490 \\
\hline Hypertension & $448(80.0)$ & $121(77.1)$ & $0.049^{*}$ \\
\hline Liver disease & $123(22.0)$ & $47(29.9)$ & 0.999 \\
\hline Fluid electrolyte & $343(61.3)$ & $96(61.1)$ & $<0.001^{*}$ \\
\hline Ventilator use $n,(\%)$ & 255 & 119 & \\
\hline No & $305(45.5)$ & $38(75.8)$ & $119(24.2)$ \\
\hline Yes & $255(54.5)$ & & \\
\hline
\end{tabular}

Table.1 Baseline characteristics between CRRT Unreceived /Received Group(continued)

\begin{tabular}{|llll|}
\hline Characteristics & CRRT Unreceived & CRRT Received & p value \\
\hline Vasopressor use n,(\%) & 374 & 155 & $<0.001^{*}$ \\
\hline No & $186(33.2)$ & $2(1.3)$ & \\
Yes & $374(66.8)$ & $155(98.7)$ & \\
\hline SOFA & $8.00(6.00,10.00)$ & $11.00(9.00,12.00)$ & $<0.001^{*}$ \\
\hline 28-Days Mortality n,(\%) & & & 0.009 \\
\hline No & $307(54.8)$ & $67(42.7)$ & \\
\hline Yes & $253(45.2)$ & $90(57.3)$ & \\
\hline
\end{tabular}

${ }^{*} \mathrm{P}$-value is less than 0.05

Table.2 Result of effects between two submodels and the Joint Model 


\begin{tabular}{|c|c|c|c|c|c|c|c|c|c|}
\hline \multirow[t]{2}{*}{ Parameter } & \multicolumn{3}{|l|}{$\operatorname{cox}$} & \multicolumn{3}{|l|}{ LME } & \multicolumn{3}{|l|}{ JM } \\
\hline & $\begin{array}{l}\text { Parameter } \\
\text { Estimate }\end{array}$ & SE & $\begin{array}{l}\mathrm{P} \\
\text { value }\end{array}$ & $\begin{array}{l}\text { Parameter } \\
\text { Estimate }\end{array}$ & SE & $\begin{array}{l}\mathrm{P} \\
\text { value }\end{array}$ & $\begin{array}{l}\text { Parameter } \\
\text { Estimate }\end{array}$ & SE & $\begin{array}{l}P \\
\text { value }\end{array}$ \\
\hline $\begin{array}{l}\text { CRRT Treatment } \\
\text { Effect on 28-Days } \\
\text { Survival }\end{array}$ & 0.176 & 0.141 & 0.213 & / & / & / & 0.148 & 0.145 & 0.307 \\
\hline $\begin{array}{l}\text { CRRT Treatment } \\
\text { Effect on } \\
\text { Log(Lactate) }\end{array}$ & / & / & / & 0.049 & 0.018 & 0.008 & 0.041 & 0.013 & 0.002 \\
\hline $\begin{array}{l}\text { Log(Lactate) Effect } \\
\text { on 28-Days Survival }\end{array}$ & I & I & / & / & I & / & 1.755 & 0.625 & 0.005 \\
\hline
\end{tabular}

Cox: Cox Proportional Hazards Model; LME: Linear Mixed Effect Model; JM: Joint Model

LME submodel independents includes: CRRT use, time of lactate level, CRRT*Time, NLR, SOFA Group and age.

Cox submodel was adjusted by: Age, ethnicity, RDW, NA, MG, PHOS, CR, UO, liver disease status, ventilator use, vasopressor use, and SOFA score.

\section{Figures}

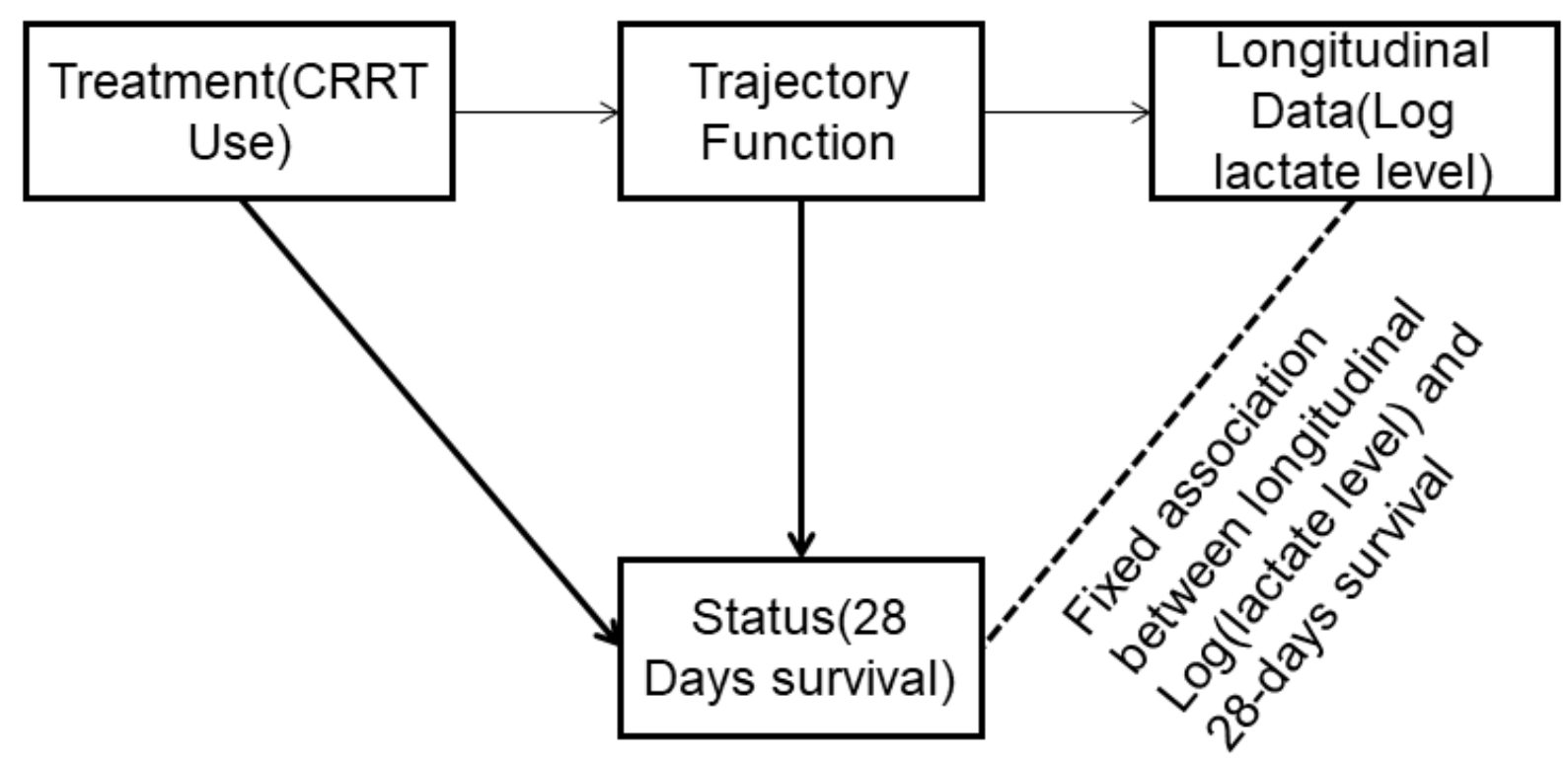

Figure 1 
(a)

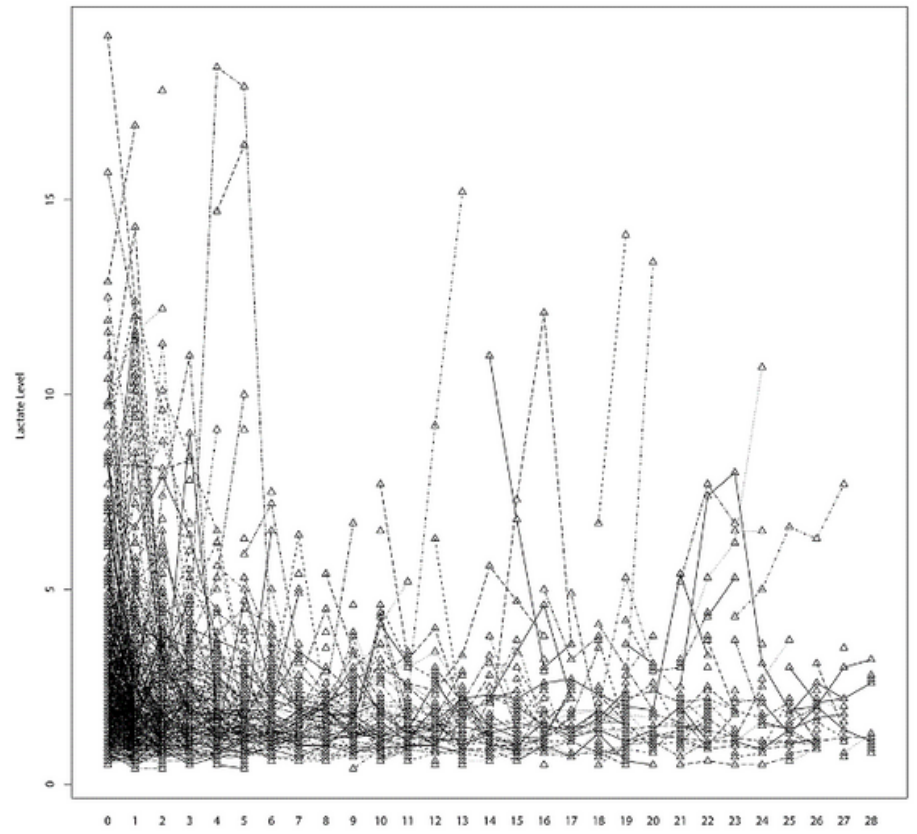

Dors (b)

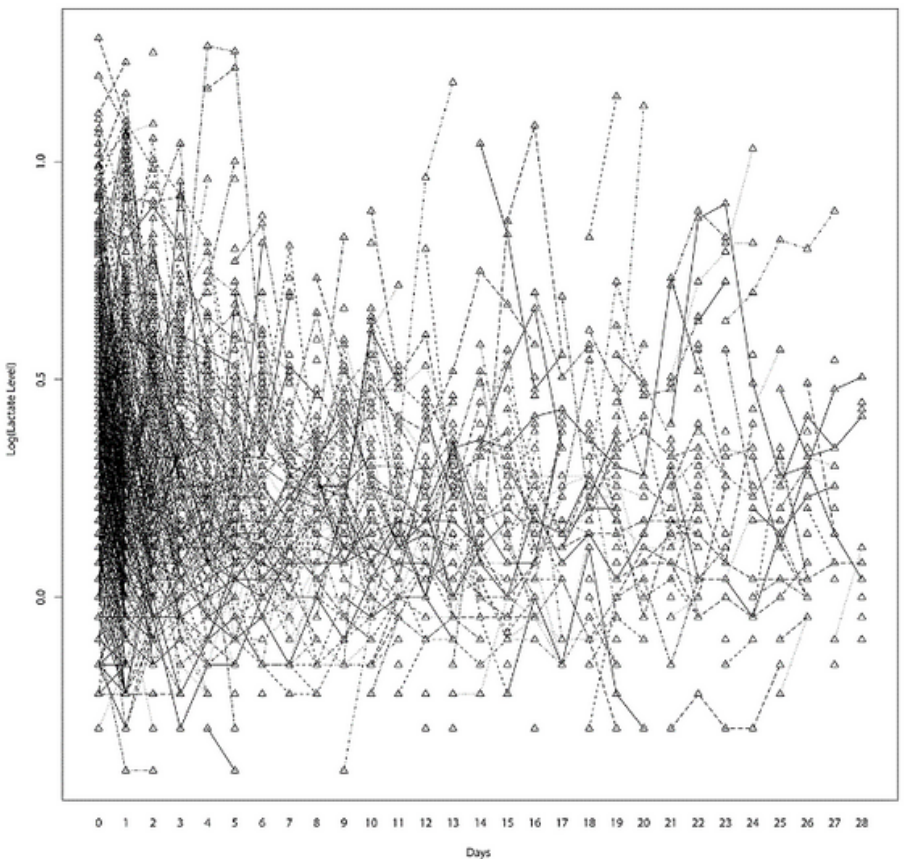

Figure 2

The trajectory record of 3661 lactate value (a) and Log(Lactate) value (b). Each line stands for a trajectory for one patient, there are 717 lines. Figure.2 (a) and (b) represents the lactate change of 717 patients involved in the study. 


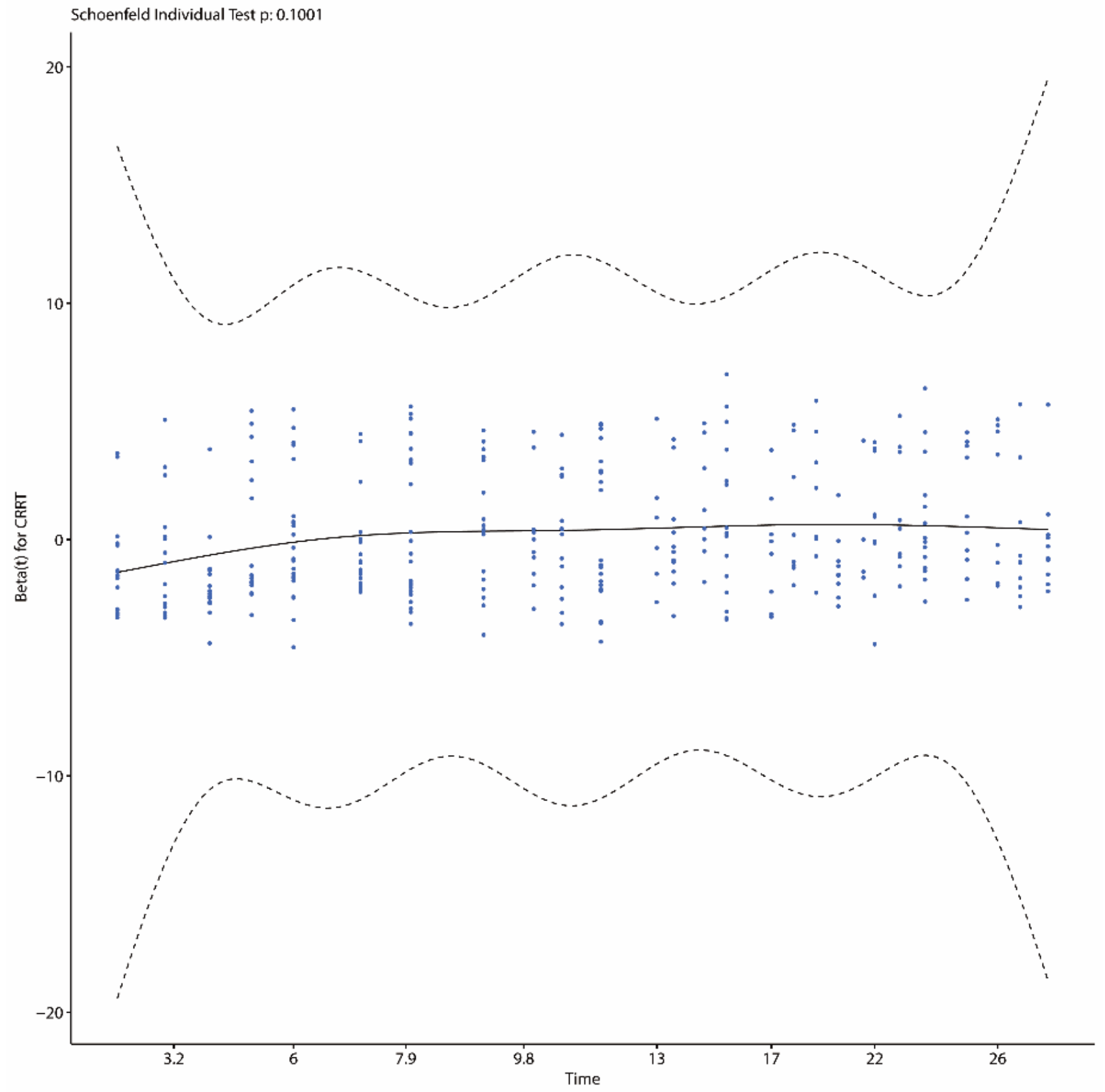

Figure 3

Schoenfeld Residuals Test result of CRRT in Cox regression analysis. The p-value of Schoenfeld Residuals Test result is larger than 0.05 which indicated that CRRT is not a time dependent variable and can be analyzed by Cox Proportional Hazards Model. 